\title{
A RATE OF CONVERGENCE RESULT FOR THE SUPER-CRITICAL GALTON-WATSON PROCESS
}

\author{
C. C. HEYDE, Australian National University
}

Let $Z_{0}=1, Z_{1}, Z_{2}, \cdots$ denote a super-critical Galton-Watson process whose non-degenerate offspring distribution has probability generating function $F(s)=\sum_{j=0}^{\infty} s^{j} P\left(Z_{1}=j\right), 0 \leqq s \leqq 1$, where $1<m=E Z_{1}<\infty$. The GaltonWatson process evolves in such a way that the generating function $F_{n}(s)$ of $Z_{n}$ is the $n$th functional iterate of $F(s)$. The convergence problem for $Z_{n}$, when appropriately normed, has been studied by quite a number of authors; for an ultimate form see Heyde [2]. However, no information has previously been obtained on the rate of such convergence. We shall here suppose that $E Z_{1}^{2}<\infty$ in which case $W_{n}=m^{-n} Z_{n}$ converges almost surely to a non-degenerate random variable $W$ as $n \rightarrow \infty$ (Harris [1], p. 13). It is our object to establish the following result on the rate of convergence of $W_{n}$ to $W$.

Theorem. Let $\operatorname{var} Z_{1}=\sigma^{2}<\infty$. As $n \rightarrow \infty, m^{n / 2}\left(W-W_{n}\right)$ converges in distribution. The limit law is given by the characteristic function relation

$$
\lim _{n \rightarrow \infty} E\left[\exp \left\{i t m^{n / 2}\left(W-W_{n}\right)\right\}\right]=E\left[\exp \left\{-\frac{1}{2} t^{2} \sigma^{2}\left(m^{2}-m\right)^{-1} W\right\}\right] .
$$

Proof. Firstly, we take $r>n$ and consider

$$
\begin{aligned}
E[ & \left.\exp \left\{i t m^{n / 2}\left(W_{r}-W_{n}\right)\right\}\right] \\
& =\sum_{j=0}^{\infty} E\left[\exp \left\{i t m^{n / 2}\left(m^{-r} Z_{r}-m^{-n} Z_{n}\right)\right\} \mid Z_{n}=j\right] P\left(Z_{n}=j\right) \\
& =\sum_{j=0}^{\infty} \exp \left\{-i t m^{-n / 2} j\right\}\left[E\left[\exp \left\{i t m^{n / 2-r} Z_{r-n}\right\}\right]\right]^{j} P\left(Z_{n}=j\right) \\
& =E\left[\exp \left\{-i t m^{-n / 2}\right\} E\left[\exp \left\{i t m^{n / 2-r} Z_{r-n}\right\}\right]\right]^{Z_{n}} .
\end{aligned}
$$

Next, we let $r \rightarrow \infty$ in (1) keeping $n$ fixed. Given $\varepsilon>0$ we can choose $N$ so large that $\sum_{j=N+1}^{\infty} P\left(Z_{n}=j\right)<\varepsilon$. Also, since $W_{r-n}$ converges in distribution to $W, E\left[\exp \left\{i t m^{n / 2-r} Z_{r-n}\right]\right]$ converges to $E\left[\exp \left\{i t m^{-n / 2} W\right\}\right]$, uniformly in any finite $t$ interval, so we can find $R$ so large that

Received 2 January 1970. 


$$
\left|E\left[\exp \left\{i t m^{n / 2-r} Z_{r-n}\right\}\right]-E\left[\exp \left\{i t m^{-n / 2} W\right\}\right]\right|<\varepsilon
$$

for $r>R$. Consequently,

$$
\begin{aligned}
& \sum_{j=0}^{\infty} \exp \left\{-i t m^{-n / 2} j\right\}\left[E\left[\exp \left\{i t m^{n / 2-r} Z_{r-n}\right\}\right]\right]^{j} P\left(Z_{n}=j\right) \\
= & \sum_{j=0}^{\infty} \exp \left\{-i t m^{-n / 2} j\right\}\left[E\left[\exp \left\{i t m^{-n / 2} W\right\}\right]\right]^{j} P\left(Z_{n}=j\right) \\
+ & \sum_{j=0}^{R} \exp \left\{-i t m^{-n / 2} j\right\}\left\{\left[E\left[\exp \left\{i t m^{n / 2-r} Z_{r-n}\right\}\right]\right]^{j}-\left[E\left[\exp \left\{i t m^{-n / 2} W\right\}\right]\right]^{j}\right\} P\left(Z_{n}=j\right) \\
+ & \sum_{j=R+1}^{\infty} \exp \left\{-i t m^{-n / 2} j\right\}\left\{\left[E\left[\exp \left\{i t m^{n / 2-r} Z_{r-n}\right\}\right]\right]^{j}-\left[E\left[\exp \left\{i t m^{-n / 2} W\right\}\right]\right]^{j}\right\} P\left(Z_{n}=j\right),
\end{aligned}
$$

and

$$
\begin{gathered}
\sum_{j=R+1}^{\infty} \exp \left\{-i t m^{-n / 2} j\right\}\left\{\left[E\left[\exp \left\{i t m^{n / 2-r} Z_{r-n}\right\}\right]\right]^{j}-\left[E\left[\exp \left\{i t m^{-n / 2} W\right\}\right]\right]^{j}\right\} P\left(Z_{n}=j\right) \mid \\
\leqq 2 \sum_{j=R+1}^{\infty} P\left(Z_{n}=j\right) \leqq 2 \varepsilon,
\end{gathered}
$$

while for $r>R$,

$$
\begin{aligned}
& \mid \sum_{j=0}^{R} \exp \left\{i t m^{-n / 2} j\right\}\left\{\left[E\left[\exp \left\{i t m^{n / 2-r} Z_{r-n}\right\}\right]\right]^{j}-\left[E\left[\exp \left\{\text { itm }^{-n / 2} W\right\}\right]\right]^{j}\right\} P\left(Z_{n}=j\right) \mid \\
& \leqq \sum_{j=0}^{R}\left|\left[E\left[\exp \left\{i t m^{n / 2-r} Z_{r-n}\right\}\right]\right]^{j}-\left[E\left[\exp \left\{i t m^{-n / 2} W\right\}\right]\right]^{j}\right| P\left(Z_{n}=j\right) \\
& \quad \leqq \varepsilon \sum_{j=0}^{R} j P\left(Z_{n}=j\right) \leqq \varepsilon m^{n}
\end{aligned}
$$

since $E Z_{n}=m^{n}$. However, $\varepsilon$ is arbitrary and $n$ fixed so that

$$
E\left[\exp \left\{i t m^{n / 2}\left(W-W_{n}\right)\right\}\right]=\lim _{r \rightarrow \infty} E\left[\exp \left\{i t m^{n / 2}\left(W_{r}-W_{n}\right)\right\}\right]
$$

$$
\begin{aligned}
& =\sum_{j=0}^{\infty} \exp \left\{-i t m^{-n / 2} j\right\}\left[E\left[\exp \left\{i t m^{-n / 2} W\right\}\right]\right]^{j} P\left(Z_{n}=j\right) \\
& =E\left[E\left[\exp \left\{i t m^{-n / 2}(W-1)\right\}\right]\right]^{Z_{n}} .
\end{aligned}
$$

Now,

$$
\begin{aligned}
& E\left[E\left[\exp \left\{i_{\text {itm }}^{-n / 2}(W-1)\right\}\right]\right]^{Z_{n}} \\
& \quad=\int_{0}^{\infty}\left[E\left[\exp \left\{i t m^{-n / 2}(W-1)\right\}\right]\right]^{x m^{n}} d P\left(m^{-n} Z_{n} \leqq x\right)
\end{aligned}
$$




$$
\begin{aligned}
=P\left(Z_{n}=0+m^{n} \log E\left[\exp \left\{i t m^{-n / 2}(W-1)\right\}\right] \int_{0}^{\infty} P\left(m^{-n} Z_{n}>x\right)\right. \\
\times\left[E\left[\exp \left\{i t m^{-n / 2}(W-1)\right\}\right]\right]^{x m n} d x,
\end{aligned}
$$

using integration by parts. Furthermore, under the hypotheses of the theorem, $W$ has mean 1 and variance $\sigma^{2} /\left(m^{2}-m\right)([1]$, p. 13) so we may expand $E\left[\exp \left\{\mathrm{itm}^{-n / 2}(W-1)\right\}\right]$ in the form

$$
E\left[\exp \left\{i t m^{-n / 2}(W-1)\right\}\right]=1-\frac{1}{2} t^{2} \sigma^{2} m^{-n}\left(m^{2}-m\right)^{-1}+o\left(t^{2} m^{-n}\right) .
$$

Thus,

$$
m^{n} \log E\left[\exp \left\{i t m^{-n / 2}(W-1)\right\}\right] \rightarrow-\frac{1}{2} t^{2} \sigma^{2}\left(m^{2}-m\right)^{-1}
$$

and

$$
\left[E\left[\exp \left\{i t m^{-n / 2}(W-1)\right\}\right]\right]^{m^{n}} \rightarrow \exp \left\{-\frac{1}{2} t^{2} \sigma^{2}\left(m^{2}-m\right)^{-1}\right\}
$$

as $n \rightarrow \infty$. Also, using (4), we have for fixed $t$ and suitably large $n$,

$$
\begin{aligned}
\left|E\left[\exp \left\{i t m^{-n / 2}(W-1)\right\}\right]\right|^{m^{n}} & <\exp \left\{-\frac{1}{2} t^{2} \sigma^{2}\left(m^{2}-m\right)^{-1}+o\left(t^{2}\right)\right\} \\
& <\exp \left\{-\frac{1}{4} t^{2} \sigma^{2}\left(m^{2}-m\right)^{-1}\right\}
\end{aligned}
$$

so that the integrand in the rightmost term of (3) is bounded in absolute value by $\exp \left\{-\frac{1}{4} t^{2} \sigma^{2} x\left(m^{2}-m\right)^{-1}\right\}$. It then follows from Fatou's lemma together with (5), (6) and since $P\left(m^{-n} Z_{n}>x\right) \rightarrow P(W>x)$ as $n \rightarrow \infty$, that

$$
\begin{array}{rl}
\lim _{n \rightarrow \infty} E & E\left[\exp \left\{i t m^{n / 2}\left(W-W_{n}\right)\right\}\right] \\
& =P(W=0)-\frac{1}{2} t^{2} \sigma^{2}\left(m^{2}-m\right)^{-1} \int_{0}^{\infty} P(W>x) \exp \left\{-\frac{1}{2} t^{2} \sigma^{2} x\left(m^{2}-m\right)^{-1}\right\} d x \\
& =\int_{0}^{\infty} \exp \left\{-\frac{1}{2} t^{2} \sigma^{2} x\left(m^{2}-m\right)^{-1}\right\} d P(W \leqq x) \\
& =E\left[\exp \left\{-\frac{1}{2} t^{2} \sigma^{2}\left(m^{2}-m\right)^{-1} W\right\}\right],
\end{array}
$$

again using integration by parts. This completes the proof of the theorem.

Unfortunately, the distribution function corresponding to the characteristic function $E\left[\exp \left\{-\frac{1}{2} t^{2} \sigma^{2}\left(m^{2}-m\right)^{-1} W\right\}\right]$ does not seem to have a useful general representation. However, in certain particular cases it may be recognised simply. 
For example, in the case of a fractional linear generating function for the offspring distribution $([1]$, pp. 9,17$)$ it is easily found to have an atom at zero and a Laplace density elsewhere.

\section{References}

[1] Harris, T. E. (1963) The Theory of Branching Processes. Springer-Verlag, Berlin.

[2] HeYDE, C. C. (1970) Extension of a result of Seneta for the super-critical Galton-Watson process. Ann. Math. Statist. 41 (in press). 